\title{
Combined Conduction And Natural Convection Cooling Of Offshore Power Cables In Porous Sea Soil
}

\author{
Ilya T’Jollyn ${ }^{1,2}$, Manly Callewaert ${ }^{3}$, Jasper Nonneman ${ }^{1,2}$, Johan Van de Wauw $^{3}$, Bernd Ameel ${ }^{1}$, Michel De Paepe Me $^{1,2}$ \\ ${ }^{1}$ Department of Flow, Heat and Combustion Mechanics \\ Ghent University \\ Sint-Pietersnieuwstraat 41 \\ Ghent, B9000, Belgium \\ ${ }^{2}$ Flanders Make, Core lab UGent-EEDT, Leuven, Belgium \\ ${ }^{3}$ Marlinks, Leuven, Belgium \\ Email: ilya.tjollyn@ugent.be
}

\begin{abstract}
The power that can be carried by offshore power cables is often restricted by the temperature limit of the materials inside the cable. It is therefore essential to predict the heat transfer behavior of the dissipated power from the cable to the environment. Offshore cables are buried in the seabed, which is a porous structure of sea soil saturated with water. Both conduction of heat through the soil, as well as natural convection due to the flow of water through the porous soil, are possible ways of heat transfer. Most cases are best described as a combination of these heat transfer effects. In this paper, a numerical model is made to predict the heat transfer from the cable to the environment by modeling the surrounding soil as a porous medium. The influence of soil parameters such as conductivity, heat capacity and permeability, as well as geometrical parameters, such as burial depth and cable diameter, are tested. An analytical expression, which can estimate the heat transfer rate for conduction dominated heat flows, is used. For convection dominated heat flows, a correlation in function of the Darcy-modified Rayleigh number is used. For heat flows which are a combination of conduction and convection effects, an algebraic summation of the thermal conductance due to convection and conduction is found not to give adequate agreement with the simulations. It is shown that an asymptotic expansion of the limiting equations for conductive and convective heat transfer rate can be used to determine the total heat flow effectively. Several soil samples in the North Sea are analyzed, and the thermal properties are used as inputs for the model. These calculations show that conduction is the main heat transfer effect and that convection has a limited effect on the heat transfer.
\end{abstract}

KEY WORDS: thermal modelling, computational fluid dynamics, porous media

\section{INTRODUCTION}

Offshore wind farms rely on submarine power cables to transport the generated power of windmills to the shore. These cables are buried in the seabed to avoid damage, mostly caused by anchors of ships and fishing activities [1]. The temperature in the cable is increased due to power dissipation. To avoid damage by overheating, this temperature and thus the power transmitted through these cables is limited. Several models have been proposed to determine the maximum current through the cable which limits the temperature of the conductors to $90{ }^{\circ} \mathrm{C}$ [2], [3]. The maximum current is highly dependent on the heat transfer in and outside the cable.

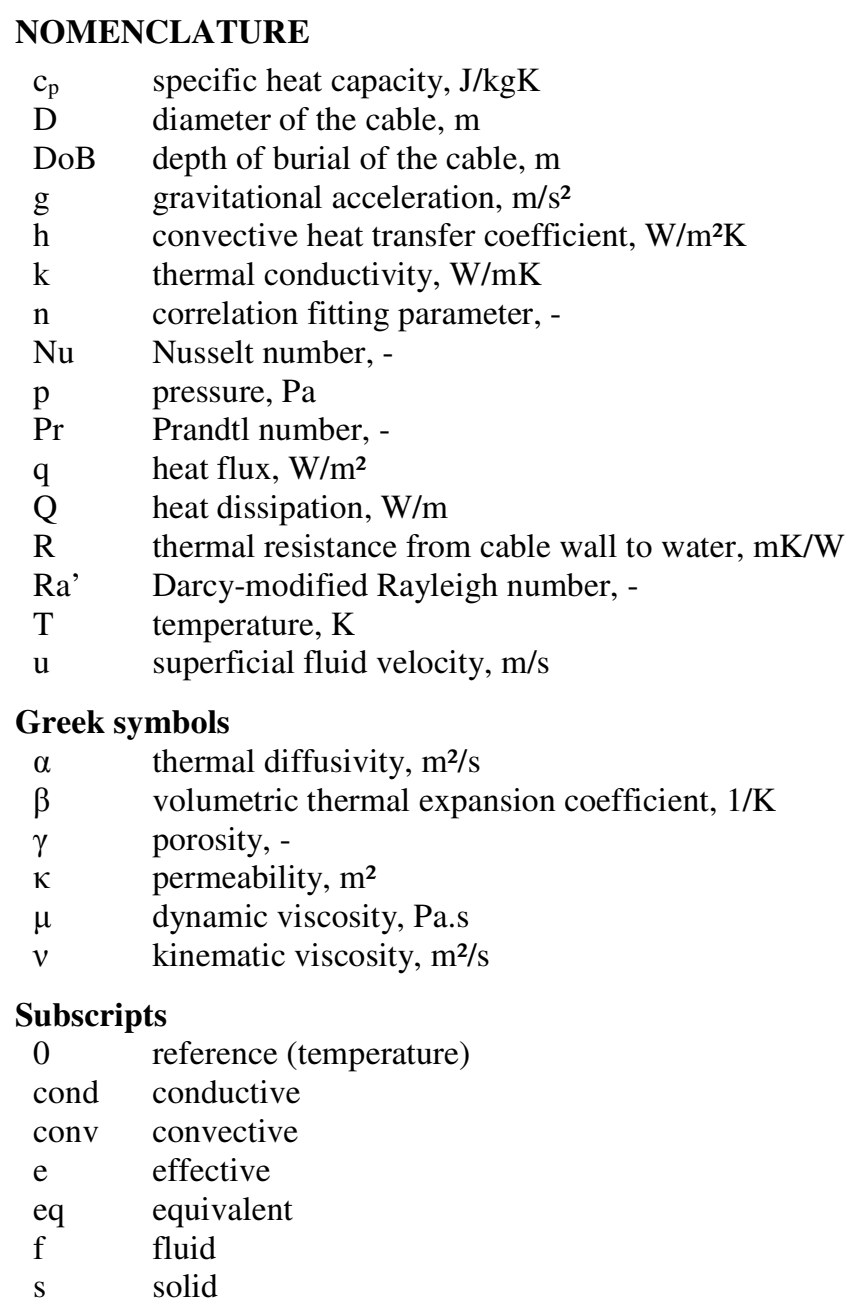

Hughes et al. did numerical simulations on the heat transfer problem and indicated that the previous modeling and simulation efforts might underestimate the heat transfer from the cable, since only conduction of heat through the soil was considered [4]. The simulation of Hughes et al. analyzes the sea soil as a porous medium, which is a mixture of a solid (sediment) and a fluid (seawater). Seawater can flow through the soil, allowing for natural convective heat transfer from the cable to the seawater and thereby increasing the heat transfer rate. These simulations are validated with an experimental setup consisting of a cylindrical heater in a box filled with spherical 
glass beads, thereby mimicking a porous sea soil [5]. The simulated and experimentally determined temperature distribution showed a good visual agreement and clearly indicated the different heat transfer mechanisms (conduction vs. convection).

Natural convection from a horizontal cylinder embedded in a porous medium has been modeled and experimentally investigated by different authors. Hardee [6] and Merkin [7] used analytical solutions for the flow and energy balance to determine a correlation for the heat transfer. Fand et al. did experiments on electrically heated horizontal cylinder, surrounded by glass beads saturated with water [8]. A correlation derived from these measurements was proposed. With a highly permeable medium and a low thermal conductivity porous structure, the convective share of the heat transfer is higher than would be the case in a sea soil. None of these authors indicate a regime where conduction is dominant.

However, it is clear from the simulations of Hughes et al. [4] and the experiments by Emeana et al. [5], that there exist conduction dominated and convection dominated heat transfer situations. However, an intermediate situation also occurs, where both conduction and natural convection contribute significantly to the total heat transfer. For both the intermediate and the convection dominated situation, it is unclear how much this affects the heat transfer from the submarine cable, since it is dependent on many parameters: soil parameters (thermal conductivity, permeability) and boundary conditions (heat dissipation in the cable, cable geometry). In this paper, the models for determining heat transfer from the cable proposed in literature are described first. Next, numerical modeling is used to analyze the heat transfer from the cable to the seawater across the different heat transfer regimes by varying the influencing parameters. The results of the numerical model are used to derive a new correlation for natural convective heat transfer. A combination of an analytical model and a correlation is used to model the heat transfer for both conductive and convective heat transfer regimes, as well as the combined regime. The objective of this simulation and modelling efforts is to determine which models for heat transfer in the soil are suitable for the rating of submarine cables for different soil parameters and boundary conditions.

\section{ANALYTICAL MODELLING}

\section{Conduction Modelling}

The IEC 60287 standard, which determines the current rating of cables, uses an analytical model to determine the total thermal resistance from the conductors to the seawater on top of the sea soil [2]. Diaz et al. expanded on this modeling using an RC-network of thermal resistances and capacitors to better predict the transient behavior and validated this model with finite element simulations [3]. In both the IEC 60287 standard and the model by Diaz et al., the total thermal resistance from the cable wall to the soil is determined analytically as:

$$
R=\frac{1}{2 \pi k_{e}} \ln \left(\frac{2 D o B}{D}+\sqrt{\left(\frac{2 D o B}{D}\right)^{2}-1}\right)
$$

where $\mathrm{R}$ is the thermal resistance, $\mathrm{k}_{\mathrm{e}}$ is the effective thermal conductivity of the soil, DoB is the depth of burial from the seabed to the axis of the cable and $\mathrm{D}$ is the diameter of the cable. When the depth of burial is sufficiently larger than the cable diameter, this thermal resistance can be simplified to:

$$
R \approx \frac{1}{2 \pi k_{e}} \ln \left(\frac{4 D o B}{D}\right)
$$

\section{Natural Convection in Porous Media}

The first correlation for natural convective heat transfer from a horizontal cylinder embedded in a porous medium is derived by Hardee [6]. By using boundary-layer analysis and the integral method, the following expression is derived:

$$
N u=0.465 R^{\prime 0.5}
$$

where $\mathrm{Nu}$ is the Nusselt number and Ra' is the Darcy-modified Rayleigh number. The Nusselt number is defined as:

$$
N u=\frac{h D}{k_{e}}=\frac{q D}{\Delta T k_{e}}
$$

where $\mathrm{h}$ is the convective heat transfer coefficient, $\mathrm{q}$ is the heat flux from the cylinder and $\Delta \mathrm{T}$ is the temperature difference between the cylinder surface and the ambient fluid. The Rayleigh number is a dimensionless number that is widely used in the analysis of natural convection flows. For natural convection in porous media, this Rayleigh number is slightly modified to take into account the flow according to the Darcy law. The Darcy-modified Rayleigh number is determined by:

$$
R a^{\prime}=\frac{g \beta \Delta T D \kappa}{v \alpha_{\mathrm{eq}}}
$$

where $g$ is the gravitational acceleration, $\beta$ is the volumetric expansion coefficient of the fluid, $\kappa$ is the porous structure permeability, $v$ is the kinematic viscosity of the fluid and $\alpha_{\mathrm{eq}}$ is the equivalent thermal diffusivity based on the effective thermal conductivity and the fluid density and specific heat capacity.

Another analytical solution for the same configuration was found by Merkin [7]:

$$
N u=0.565 R a^{\prime 0.5}
$$

which differs only in the value of the first coefficient.

By fitting to experimental results, the following correlation for the Nusselt number was proposed by Fand et al. [8]:

$$
N u=0.653 R a^{\prime 0.649} \mathrm{Pr}^{0.124}
$$

where the newly introduced parameter $\mathrm{Pr}$ is the dimensionless Prandtl number. Furthermore, the exponent for the Darcymodified Rayleigh number is higher than the value of 0.5 predicted by the analytical analyses.

\section{NUMERICAL SIMULATION}

Computational fluid dynamics simulations of the problem are made using the Ansys Fluent software. The focus of this paper is on the heat transfer in the soil, which is modeled as a porous medium. Only steady-state simulations are reported on in this paper.

\section{Governing Equations}

For the flow regimes that occur due to the heating of the cable, the Darcy law can be used to determine the additional viscous losses and pressure gradient. The Darcy law is given by:

$$
\nabla p=\frac{\mu}{\kappa} u
$$




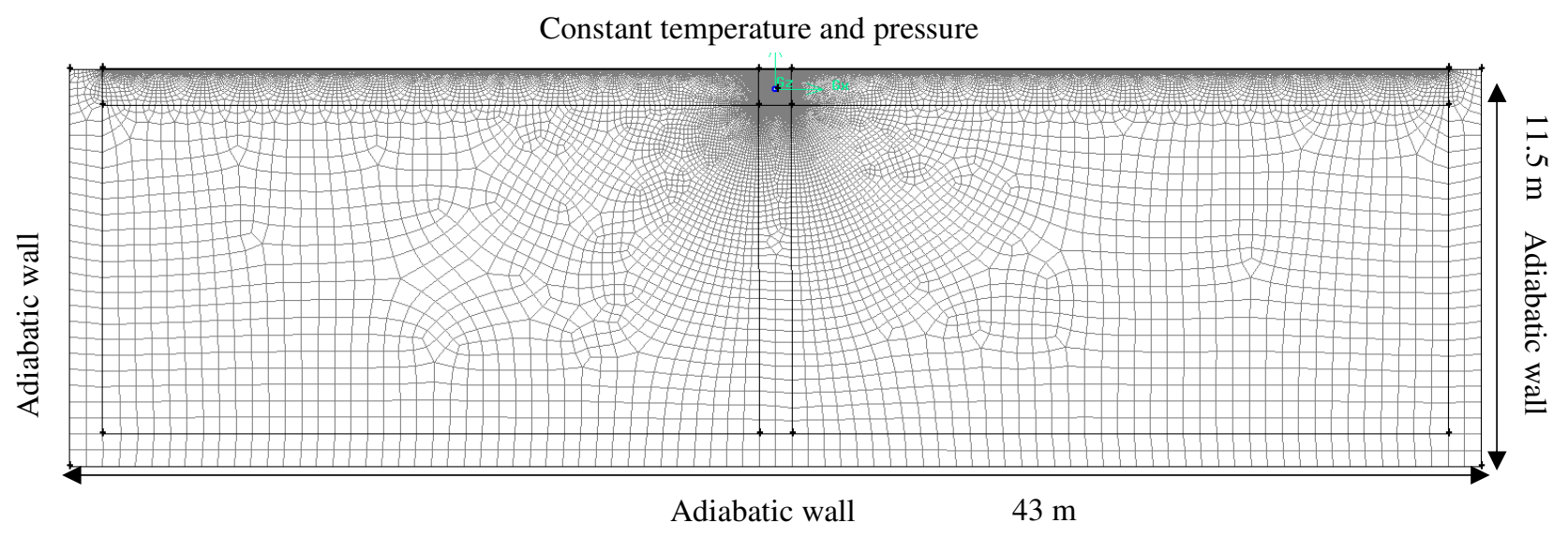

Fig. 1 Geometry and mesh of cable and sea

where $\mathrm{p}$ is the pressure, $\mu$ is the dynamic viscosity, $\kappa$ is the permeability and $u$ is the superficial fluid velocity. To account for the buoyancy forces by heating of the water, the density of the water in the simulation is evaluated as a function of temperature.

Next to the flow equations, the energy equation is solved to determine the heat transfer in the simulation domain. The thermal equilibrium model is used in the simulations, since the pore scale is several dimensions smaller than the depth of burial and the thermal conductivity of the fluid and solid are in the same order of magnitude. The energy equation in the porous soil is evaluated using the effective properties of the porous soil, which can be derived from the properties of the solid (sediment) and the liquid (water). For steady-state simulations, only the effective thermal conductivity $\mathrm{k}_{\mathrm{e}}$ is used in the energy equation, which is defined by:

$$
k_{e}=\gamma k_{f}+(1-\gamma) k_{s}
$$

where $\gamma$ is the porosity of the soil, $\mathrm{k}_{\mathrm{f}}$ is the thermal conductivity of the fluid (in this case water) and $\mathrm{k}_{\mathrm{s}}$ is the thermal conductivity of the solid (in the case sediment). Since the effective thermal conductivity is the property measured in practice and this value is used indirectly as an input for the simulation, the manner of modelling the effective thermal conductivity has no effect on the outcome of the simulations.

The second order upwind discretization scheme is used for the energy equation. Iterations are performed until the energy residual is below $10^{-9}$. There was no change in the solution when the residuals changed by another order of magnitude. . Nu turbulence model was used, as the flow velocity is very low, due to the limited permeability of the soil.. For the momentum equations, the second order upwind discretization scheme is also used. A pressure-based solver using the body-forceweighted interpolation scheme is used, since this can adequately solve flows where the buoyancy effect is important. Iterations are performed until the continuity (conservation of mass) and velocity residuals are below $10^{-4}$ (and the energy residual is below $10^{-9}$ ). There was no change in the solution when the residuals changed by another order of magnitude.

\section{Geometry and Meshing}

The problem is considered as a $2 \mathrm{D}$ problem, assuming there is little variation in the dimension along the length of the cable. The cable itself is not modeled, since the focus is on the heat transfer in the soil. The cable heating effect is generated by applying a heat flux on the circle boundary where the cable is located. A diameter of $11 \mathrm{~cm}$ is used in the simulations. The soil is modeled as a rectangular area with a depth of $11.5 \mathrm{~m}$ below the cable and a length of $43 \mathrm{~m}$. The numerical simulations have shown that taking larger areas for the soil have no significant influence on the heat transfer from the cable.

An example of the meshing is shown in Fig. 1. Quadrilateral elements are used to generate the mesh. The elements close to the cable wall are generated with a boundary layer mesh (shown in blue on Fig. 2), which is suitable for solving the boundary layers which can occur during natural convective heat transfer. A smaller meshing size is used close to the cable, where the highest thermal and pressure gradients will occur. The meshing size increases gradually when moving away from the cable.

The error on the simulation due to finite grid discretization is estimated by assessing two grids: a standard (fine) grid and a coarse grid with around four times less mesh faces. With both grids, a steady-state calculation is done to determine the temperature difference between cable wall and water. A heating load of $100 \mathrm{~W} / \mathrm{m}$ is applied to the cable wall. The temperature difference for the fine and coarse grid is respectively 25.04524 $\mathrm{K}$ and $25.06435 \mathrm{~K}$. This results in an absolute difference of $0.01911 \mathrm{~K}$ or a relative difference of $0.08 \%$. According to Roache, a good estimation of the maximum error due to the finite grid discretization is given by the grid convergence index

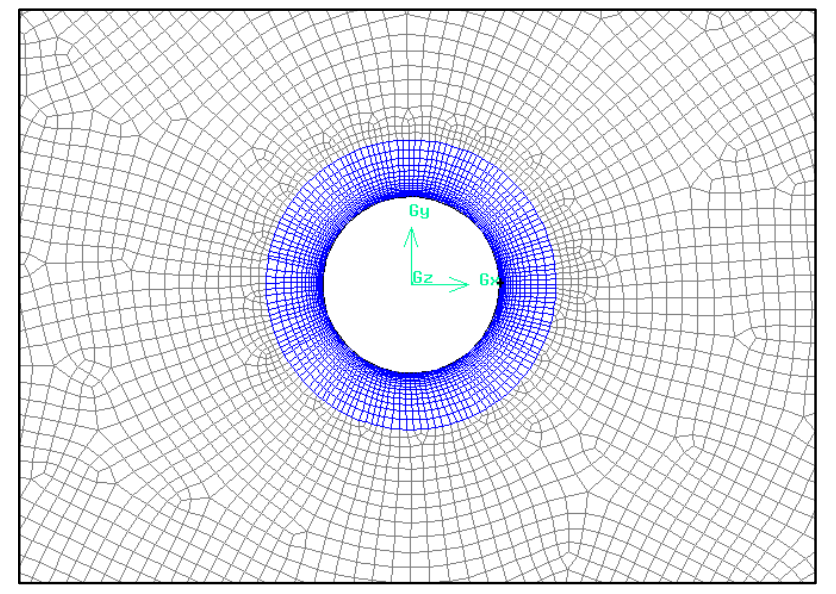

Fig. 2 Close-up of the boundary layer mesh close to the cable 
(GCI) [9]. For a 2D-simulation, if the ratio of the number of cells between the fine and the coarse grid is four, the GCI on the fine grid is equal to the relative error. This indicates that using the fine grid results in errors due to grid discretization which are most likely smaller than $0.08 \%$.

\section{Boundary Conditions}

The heat dissipation in the cable is varied to determine the effect on the heat transfer. A uniform heat flux $q$ is applied to the cable wall, which is related to the heat dissipation per meter cable Q as:

$$
q=\frac{Q}{\pi D}
$$

To assess the influence of the type of the boundary condition, simulations are also done with a constant temperature boundary. In these simulations, the cable wall temperature is set to a fixed temperature and the heat flux is determined by the numerical simulation.

At the top, a layer of a constant temperature of $20^{\circ} \mathrm{C}$ is added that represents the seawater on top of the sea soil. The top boundary is implemented as by fixing the total pressure and the temperature of the water. The other boundaries are implemented as adiabatic walls, indicating no heat or water can pass through these boundaries.

\section{Material Properties}

For the seawater, the properties in Table 1 are used. To account for the buoyancy effect, the density is implemented as a linear function of temperature, as shown in the equation below:

$$
\rho_{f}=\rho_{0}\left(1-\beta\left(T-T_{0}\right)\right)
$$

The reference temperature $\mathrm{T}_{0}$, density at the reference temperature $\rho_{0}$ and the thermal expansion coefficient can be found in Table 1.

The soil properties affecting the heat transfer are the effective thermal conductivity and the permeability. A value of $2.15 \mathrm{~W} / \mathrm{mK}$ is used for the soil thermal conductivity in the simulations, which is a representative value for sea soils (values range from $0.8 \mathrm{~W} / \mathrm{mK}$ to $3.11 \mathrm{~W} / \mathrm{mK}$ ) [4]. The permeability is varied from $10-12 \mathrm{~m}^{2}$ to $10-8 \mathrm{~m}^{2}$. The lower limit represents soils with a low permeability (e.g. clay) where the flow does not affect the heat transfer up. The higher limit is representative for highly coarse and permeable sand soils.

Table 1. Water properties used in the numerical simulations

\begin{tabular}{lrl} 
Property & Value & Unit \\
\hline $\mathrm{k}_{\mathrm{f}}$ & 0.6 & $\mathrm{~W} / \mathrm{mK}$ \\
$\mathrm{c}_{\mathrm{p}, \mathrm{f}}$ & 4182 & $\mathrm{~J} / \mathrm{kgK}$ \\
$\rho_{0}$ & 998.8 & $\mathrm{~kg} / \mathrm{m}^{3}$ \\
$\mathrm{~T}_{0}$ & 293.15 & $\mathrm{~K}$ \\
$\beta$ & 0.000205 & $1 / \mathrm{K}$ \\
$v$ & 0.001 & $\mathrm{~m}^{2} / \mathrm{s}$
\end{tabular}

\section{SIMULATION RESULTS AND DISCUSSION}

The parameters that have the biggest influence on the convective heat transfer are the soil permeability and the temperature difference between cable wall and water. The permeability is varied between $10^{-12} \mathrm{~m}^{2}$ and $10^{-8} \mathrm{~m}^{2}$, with the chosen thirteen values logarithmically spaced. The heat load is evaluated at $25 \mathrm{~W} / \mathrm{m}$, as well as at $50 \mathrm{~W} / \mathrm{m}$ to $400 \mathrm{~W} / \mathrm{m}$ in steps of $50 \mathrm{~W} / \mathrm{m}$. Another kind of simulations is also performed, where the temperature at the cable wall is set and the heat flow from the cable is monitored. In these simulations the temperature difference between cable wall and water is set to a value ranging from $2.5^{\circ} \mathrm{C}$, plus from $5{ }^{\circ} \mathrm{C}$ to $40{ }^{\circ} \mathrm{C}$ in steps of $5{ }^{\circ} \mathrm{C}$. The convective heat transfer rate is assumed to be independent of the depth of burial. To test this hypothesis, three different values for the burial depth are used in the simulations $(40 \mathrm{~cm}, 60 \mathrm{~cm}$ and $500 \mathrm{~cm}$ ). All these variations result in 585 data points in total (the simulations of $500 \mathrm{~cm}$ burial depth were only done for a constant temperature boundary condition).

A select number of the 585 data points are shown in Fig. 3, where the cable wall temperature difference (with the water) is plotted as a function of the soil permeability. The simulations show that for low permeability $\left(<10^{-11} \mathrm{~m}^{2}\right)$, there is no influence of convection. At higher permeability, the temperature difference decreases due to the convective heat flow. The influence of the burial depth is clear for the conductive heat transfer dominated cases, but the influence becomes smaller for convection dominated heat transfer. Furthermore, the temperature difference varies linearly with the heat load for conduction dominated cases, but this no longer holds true if convection has an influence on the heat transfer. The temperature fields for both a conduction and a convection dominated heat transfer condition are shown in Fig. 4.

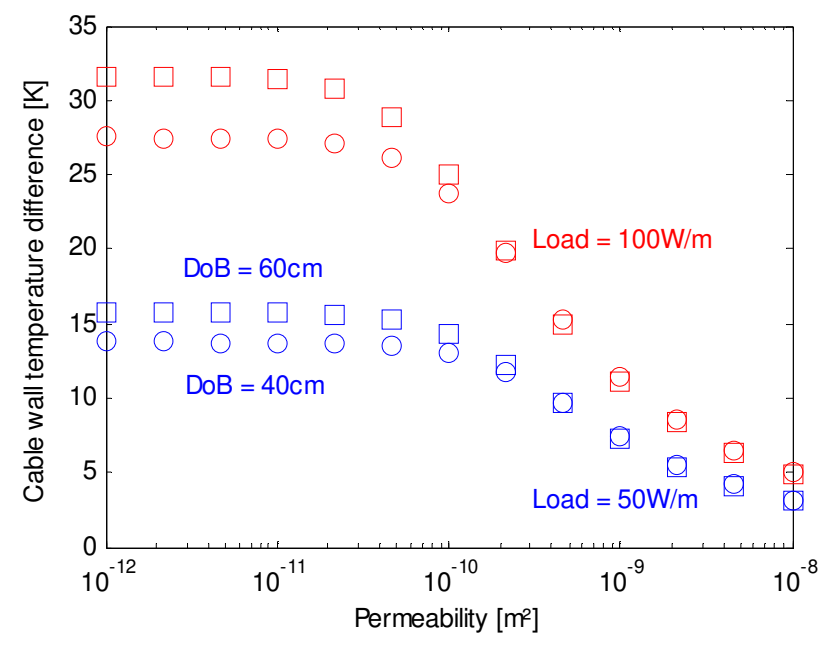

Fig. 3 Cable wall temperature difference in function of permeability

The Nusselt number is shown for all the simulations as a function of the Darcy-modified Rayleigh number in Fig. 5. The Nusselt number for conduction $\mathrm{Nu}_{\text {cond }}$, shown in green for the three different burial depths, is defined as a function of the analytical solution in equation (2):

$$
N u_{\text {cond }}=\frac{1}{\pi k_{e} R}=\frac{2}{\ln \left(\frac{4 D o B}{D}\right)}
$$

For low Rayleigh numbers, the simulations agree well with the analytical conduction model. For higher Rayleigh numbers, the Nusselt number increases due to convection. At high Rayleigh numbers, the Nusselt number varies with the Darcy-modified Rayleigh number with a power 0.5 , as in equation (6). The convective Nusselt number $\mathrm{Nu}_{\text {conv }}$ is shown in red on Fig. 5. 

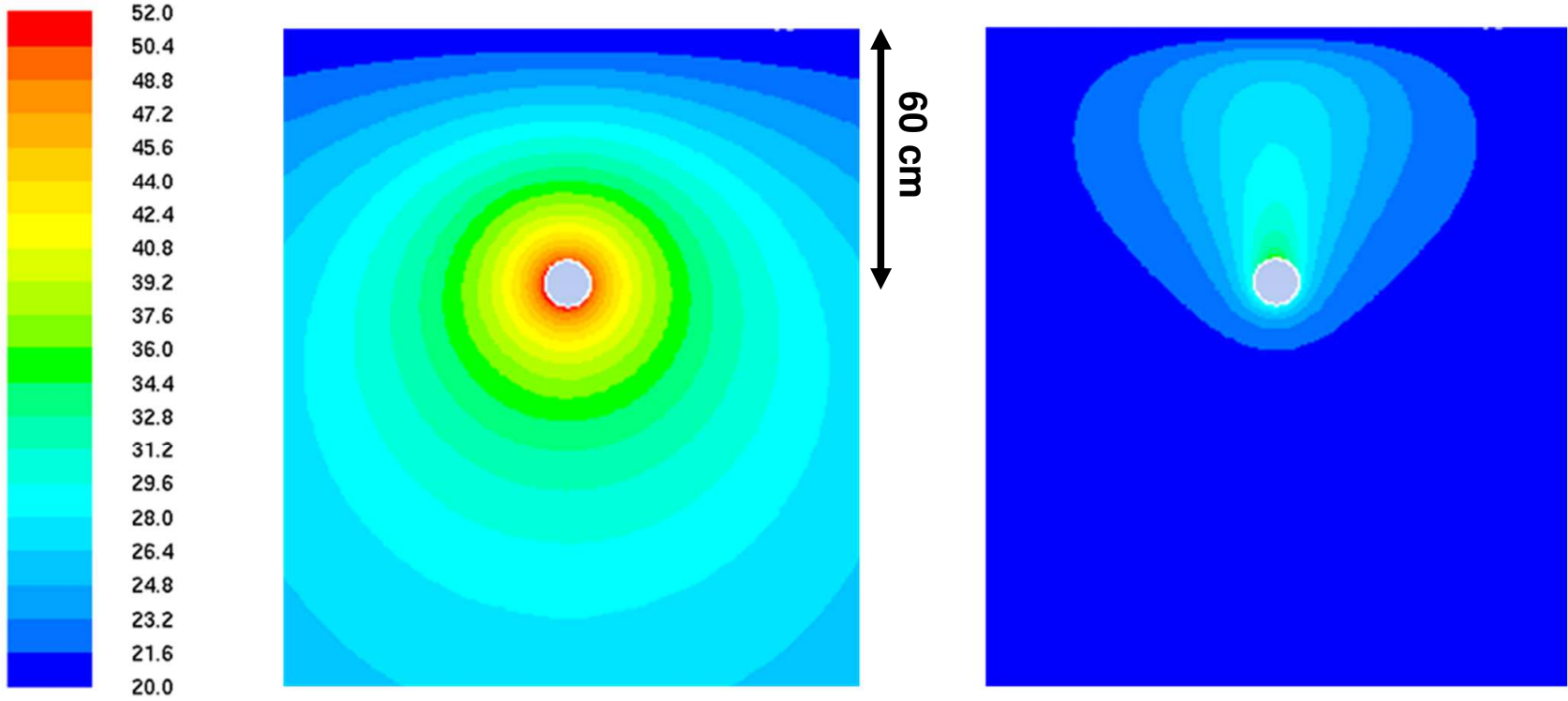

Fig. 4 Simulated temperature field around cable for a soil with low $\left(10^{-11} \mathrm{~m}^{2}\right.$, left $)$ and high $\left(10^{-9} \mathrm{~m}^{2}\right.$, right $)$ permeability

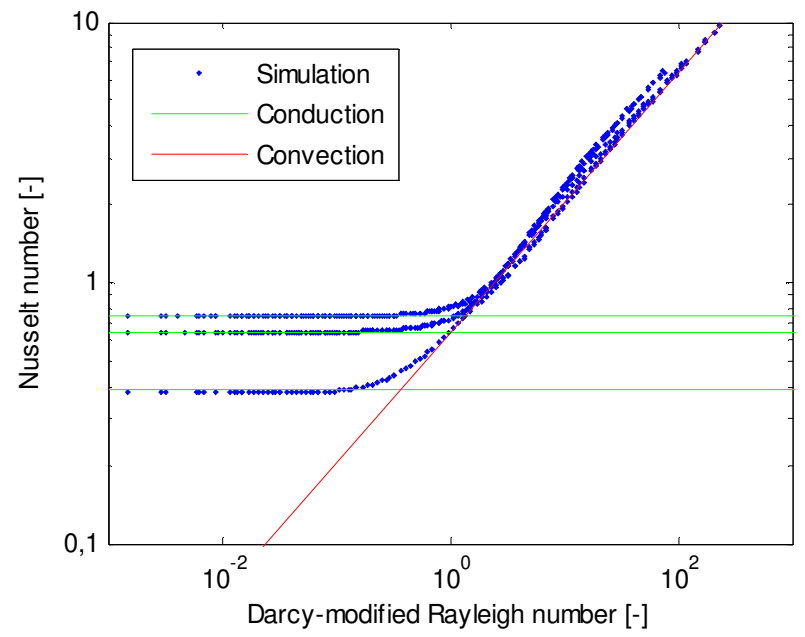

Fig. 5 Nusselt number as a function of the Darcy-modified Rayleigh number with equations for conductive and convective heat transfer for three different DoB

A summation of both Nusselt numbers does not yield good result for the transition region from conduction to convection dominated conditions. As proposed and shown by Churchill \& Usagi, the following expression can be used for transport processes which vary uniformly between two limiting solutions (in this case purely conductive and purely convective heat transfer) [10]:

$$
N u=\left(N u_{\text {cond }}^{n}+N u_{\text {conv }}^{n}\right)^{1 / n}
$$

In this equation the parameter $\mathrm{n}$ can optimized for the best fit. Churchill \& Usagi propose to choose the closest integer value for $\mathrm{n}$ for simplicity since the resulting equation is quite insensitive to this parameter. For these simulations, a value for $\mathrm{n}$ of five is determined and the resulting correlation is shown in Fig. 6. With this correlation, all the simulated temperature differences are predicted within an error of $13 \%$.
A validation simulation set is done to evaluate if the correlation takes into account all effects properly. A full factorial design is done on five input parameters: effective thermal conductivity, permeability, burial depth, cable diameter and heat dissipation. Two levels (a high and a low level, shown in Table 2) for each parameter are used, resulting in 32 additional simulations. All these additional simulated temperature difference fall within the same error band of $13 \%$, indicating that the effect of thermal conductivity and diameter and their interaction effects with the other parameters are also predicted accurately.

Table 2. Full factorial design for validation simulation set

\begin{tabular}{lrll} 
Property & Low level & High level & Unit \\
\hline $\mathrm{k}_{\mathrm{e}}$ & 1.536 & 3.603 & $\mathrm{~W} / \mathrm{mK}$ \\
$\kappa$ & $10^{-10}$ & $10^{-9}$ & $\mathrm{~m}^{2}$ \\
$\mathrm{DoB}$ & 20 & 100 & $\mathrm{~cm}$ \\
$\mathrm{D}$ & 10 & 30 & $\mathrm{~cm}$ \\
$\mathrm{Q}$ & 50 & 200 & $\mathrm{~W} / \mathrm{m}$
\end{tabular}

\section{EFFECT ON CABLES IN BELGIAN NORTH SEA}

From the simulation results, it is clear that the effect of convection can be dominating in the heat transfer from the cable to the seawater, depending on the soil characteristics. Soil samples of ten locations in the Belgian North Sea were gathered and their soil properties determined. The effective thermal conductivity ranged from $2.091 \mathrm{~W} / \mathrm{mK}$ to $2.799 \mathrm{~W} / \mathrm{mK}$, while the permeability ranged from $9.7210^{-13} \mathrm{~m}^{2}$ to $4.6210^{-11} \mathrm{~m}^{2}$. Distributed temperature sensing inside a power cable in the North Sea showed that the temperature difference between the cable wall and the seawater does not exceed $20^{\circ} \mathrm{C}$. For a cable of $21 \mathrm{~cm}$ in diameter, buried up to $2 \mathrm{~m}$ in the soil, it is calculated how much higher the cable wall temperature would be if no convection would occur. The maximum increase in temperature is equal to $3.4^{\circ} \mathrm{C}$ or $17 \%$. This indicates that conduction is the 


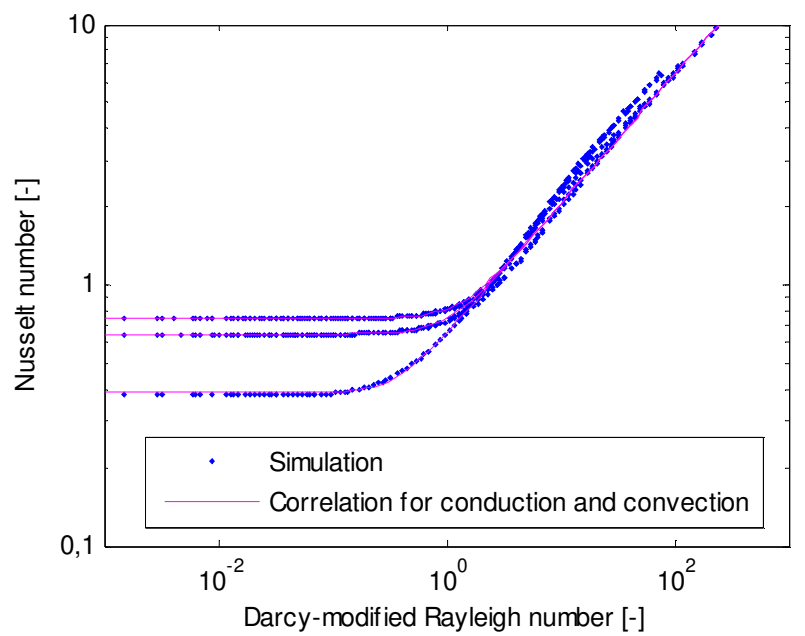

Fig. 6 Nusselt number as a function of the Darcy-modified Rayleigh number with the determined correlation for conduction and convection for three different DoB

main heat transfer mechanism and that the maximum deviation made by an assumption of purely conductive heat transfer for the soils in the North Sea would be equal to $3.4^{\circ} \mathrm{C}$. When taking into account the temperature differences between the cable wall and the cable core (which can go up to $50^{\circ} \mathrm{C}$ ), this decrease of $3.4^{\circ} \mathrm{C}$ only accounts for about $5 \%$ of the temperature difference between cable core and ambient. Therefore, rating the maximal current of submarine power cables in the Belgian North Sea using the existing methods that only take into account conductive heat transfer is on the safe side, but it is not a very significant underestimation. In other regions, with more coarse and permeable sediment types (gravel) such as some locations on the coast of the United Kingdom, taking into account convection could increase the maximal current rating and thus the maximum power that can be transferred through the cable.

\section{CONCLUSION}

The maximal current rating of submarine power cables is based on thermal modeling of the heat transfer from the cable to the seawater. The current practice is to use methods which only take conduction into account, neglecting the convective heat transfer.

Simulations are performed to determine the heat transfer from a cable, which is simplified to a cylinder with a uniform heat flux or temperature at the cylinder wall. The simulations show that convection and conduction can both be dominant, dependent on the material properties of the soil and on the geometry. A Darcy-modified Rayleigh number correlation is used for convection dominated heat transfer. To adequately model the conditions where both conductive and convective heat transfer are significant, an expression taking into account both effects is determined, based on the proposal of Churchill \& Usagi for transport processes uniformly varying between two limiting solutions. All 617 numerical simulation results are predicted by the proposed expression with a maximum error of $13 \%$ on the wall temperature difference.

For cables in the Belgian North Sea, the highest decrease in cable core temperature due to convective effects is determined using the derived expression. Based on soil property measurements, the highest expected decrease is estimated as $3.4^{\circ} \mathrm{C}$, which will have a limited effect on the maximum current rating. In other locations, for very coarse and highly permeable soils, convective effects can potentially be taken into account to achieve a higher current rating.

\section{Acknowledgements}

This work features results gathered during the course of the $R \& D$ project 'A novel method for continuous monitoring of the burial depth of offshore power cables to minimize cable failure risks' by Marlinks (project number HBC.2016.0761), funded by the Flemish agency for entrepreneurship and innovation (VLAIO). The financial support is gratefully acknowledged.

\section{References}

[1] M. Kordahi, S. Shapiro and G. Lucas, "Trends in submarine cable system faults," in Submarine Optical Conference, Baltimore, 2007.

[2] Calculation of the Current Ratings, IEC Standard 60287-1 and IEC 60287-2, 2001.

[3] M. Diaz-Aguiló, F. De Leon, S. Jazebi and M. Terracciano, "Ladder-type soil model for dynamic thermal rating of underground power cables," IEEE Power and Energy Technology Systems Journal, vol. 1, pp. 21-30, 2014.

[4] T. Hughes, T. Henstock, J. Pilgrim, J. Dix, T. Gernon and C. Thompson, "Effect of sediment properties on the thermal performance of submarine HV cables," IEEE Transactions on Power Delivery, vol. 30, no. 6, pp. 2443-2450, 2015.

[5] C. Emeana, T. Hughes, J. Dix, T. Gernon, T. Henstock, C. Thompson and J. Pilgrim, "The thermal regime around buried submarine high-voltage cables," Geophysical Journal International, vol. 206, no. 2, pp. 1051-1064, 2016.

[6] H. C. Hardee, Boundary layer solutions for natural convection in porous media, Albuquerque, New Mexico, USA: Sandia Labs, 1976.

[7] J. H. Merkin, "Free convection boundary layers on axisymmetric and two-dimensional bodies of arbitrary shape in a saturated porous medium," International Journal of Heat and Mass Transfer, vol. 22, pp. 14611462, 1979.

[8] R. M. Fand, T. E. Steinberger and P. Cheng, "Natural convection heat transfer from a horizontal cylinder embedded in a porous medium," International Journal of Heat and Mass Transfer, vol. 29, no. 1, pp. 119-133, 1986.

[9] P. J. Roache, "Quantification of uncertainty in computational fluid dynamics.," Annual Review of Fluid Mechanics, vol. 29, no. 1, pp. 123-160, 1997.

[10] S. W. Churchill and R. Usagi, "A General Expression for the Correlation of Rates of Transfer and Other Phenomena," AIChE Journal, vol. 18, no. 6, pp. 1121$1128,1972$. 